\title{
Simultaneous carriage of multiple genotypes of Staphylococcus aureus in children
}

\begin{abstract}
Correspondence
Kanokporn Mongkolrattanothai kmongkol@uic.edu
\end{abstract}

Received 3 September 2010 Accepted 6 November 2010

\author{
Kanokporn Mongkolrattanothai, ${ }^{1}$ Barry M. Gray, ${ }^{1}$ Peggy Mankin, ${ }^{1}$ \\ Amy B. Stanfill, ${ }^{2}$ Richard H. Pearl, ${ }^{2}$ Lizabeth J. Wallace ${ }^{2}$ \\ and Ravindra K. Vegunta ${ }^{2} \dagger$
}
${ }^{1}$ Department of Pediatrics, University of Illinois College of Medicine at Peoria and the Children's Hospital of Illinois at OSF Saint Francis Medical Center, Illinois, USA
${ }^{2}$ Department of Surgery, University of Illinois College of Medicine at Peoria and the Children's Hospital of Illinois at OSF Saint Francis Medical Center, Illinois, USA

The co-existence of multiple genotypes in colonization by Staphylococcus aureus has not been fully investigated. The aim of this study was to evaluate the heterogeneity of $S$. aureus carriage in children. We evaluated 125 nasal and perianal swab samples that were positive for $S$. aureus from 76 children scheduled for elective surgery. For each sample, at least four colonies with the same or different morphotypes were selected for analysis. Multiple-locus variable-number tandem-repeat fingerprinting was used to determine the genetic relatedness and to characterize the clonality of the $S$. aureus strains. Of the 125 swabs, 91 (73\%) contained meticillin-sensitive S. aureus (MSSA), 8 (6\%) contained meticillin-resistant S. aureus (MRSA), and $26(21 \%)$ contained MSSA and MRSA simultaneously. A total of $738 \mathrm{~S}$. aureus strains were evaluated with a mean of 6 colonies (range 4-15) picked from each culture. Of the 125 swabs, 32 (26\%) samples contained two genetically distinct $S$. aureus strains and $6(5 \%)$ contained three different genotypes. Multiple $S$. aureus strains simultaneously carried by individual children were genetically unrelated to each other. We concluded that the co-existence of multiple genotypes of $S$. aureus was common. The significance of multiple carriage is yet to be determined, but this intraspecies interplay could be important to pathogenicity and virulence in $S$. aureus.

\section{INTRODUCTION}

Staphylococcus aureus is an important pathogen responsible for a wide range of clinical entities from asymptomatic colonization to invasive and life-threatening infections. Carriage of $S$. aureus has been associated with subsequent infections, especially surgical site infections (Herwaldt, 2003; Wenzel \& Perl, 1995), and a variety of other clinical conditions (Keene et al., 2005; Niven et al., 2009; Von Eiff et al., 2001). Studies have revealed that among individuals with $S$. aureus infections, the infecting strains were indeed genetically identical or related to the carriage strains (Keene et al., 2005; Kreft et al., 2001; Von Eiff et al., 2001).

Two studies have reported the carriage of multiple subtypes of meticillin-resistant S. aureus (MRSA) in sequential cultures from adult patients during their stay in an intensive care unit (Bloemendaal et al., 2009; Lim et al.,

†Present address: Banner Paediatric Specialists, Suite 301, 1432 S. Dobson Road, Mesa, AZ, USA.

Abbreviations: MLVF, multiple-locus variable-number tandem-repeat fingerprinting; MRSA, meticillin-resistant Staphylococcus aureus; MSSA, meticillin-sensitive Staphylococcus aureus.
2006). However, Bloemendaal et al. (2009) did not look at multiple colonies within the same $S$. aureus positive swabs. Therefore, it was uncertain whether these episodes were polyclonal as a result of multiple clones simultaneously colonizing patients, or acquisition of different MRSA strains over time. Lim et al. (2006) studied MRSA carriage at various body sites and found that multiple subtypes can be carried simultaneously at different sites by a single patient. Simultaneous carriage of more than one genetically distinct $S$. aureus isolate was also reported in groups of paediatric patients with certain medical conditions, such as cystic fibrosis and atopic dermatitis (Goerke et al., 2007; Lomholt et al., 2005). However, the frequency of concurrent colonization with multiple genotypes among otherwise healthy children has not been investigated.

We previously described the carriage of $S$. aureus in a cohort of 499 children scheduled for elective surgery (Vegunta et al., 2009). A total of $36 \%$ of the children carried S. aureus, of which $76 \%$ were MRSA, but surgical site infections occurred in only $2 \%$ of procedures. In the laboratory, we noticed some inconsistent phenotypic and genotypic results of $S$. aureus within a number of cultures from the same individuals, including differences in 
antimicrobial susceptibility pattern and the presence or absence of Panton-Valentine leukocidin genes and/or erm genes determined by PCR detection. This led us to look for multiple clones of $S$. aureus carried by individual patients. The aim of this work was to evaluate the genetic diversity among $S$. aureus carriage isolates with regard to the frequency of carriage by single or multiple strains.

\section{METHODS}

Sample selection. The bacterial isolates and culture swabs in this work were selected from those collected in our prospective study of MRSA colonization in 499 children scheduled for elective surgery (Vegunta et al., 2009). Nasal swabs were obtained at the presurgical outpatient visit (visit 1) and at the time of operation (visit 2); a perianal swab was also obtained at the time of the operation. There were 229 children who had nasal samples collected at both time points; 74 had at least 1 positive culture. Of these, 33 had cultures positive at only one visit, and 41 had cultures positive at both visit 1 and 2 . We chose to study the samples from these 74 children to look for co-carriage of multiple genotypes, and to examine the samples from the latter subset of 41 children to assess possible strain replacement over time. In addition, we examined $S$. aureus strains from 8 children who had positive cultures from both nasal and perianal swabs to assess the cocarriage of multiple genotypes at different body sites ( 6 were in the group of 74 children and 2 others were from the group of children who each had only 1 nasal swab obtained).

Bacterial cultures. The culture collection consisted of bacterial isolates, which were kept in egg-based media, and an aliquot of the swab cultures, which was kept in medium containing skimmed milk, tryptone, glucose and glycerin (STGG) at $-80{ }^{\circ} \mathrm{C}$ (Gray, 2002). The selected cultures were inoculated on Columbia CNA (colistinnalidixic acid) with $5 \%$ sheep blood agar plates to obtain wellseparated colonies. After $24 \mathrm{~h}$ incubation at $35{ }^{\circ} \mathrm{C}$, growth characteristics and colony morphologies (shape, size, margin, texture and degree of haemolysis) were assessed. The plates were then kept at room temperature and reassessed $24 \mathrm{~h}$ later by the same observer to increase visual recognition of different colony types. For each $S$. aureus-positive swab sample, at least four colonies with different morphotypes were selected (Coen et al., 2006). If the colonies on a plate looked alike, four colonies were randomly selected. Selective $S$. aureus agar (BBL CHROMagar Staph aureus; BD Diagnostics) and Staphaurex latex agglutination tests (Remel) were used to identify $S$. aureus. Hereafter, an individual bacterial colony collected from each swab sample is referred to as a 'strain'. All strains were subjected to further molecular analysis.

Selection of MRSA isolates. Detection of meticillin resistance was performed using the $30 \mu \mathrm{g}$ cefoxitin disc diffusion test. Using a loop, colonies were scraped from the heavy inoculum in the first quadrant of the Columbia CNA with $5 \%$ sheep blood agar plate. This approach was mainly used to increase the likelihood of MRSA detection. A suspension was prepared to a $0.5 \mathrm{McF}$ arland standard and plated on Mueller-Hinton agar. The resistance and susceptibility breakpoints for detecting meticillin resistance were $\leqslant 21$ and $\geqslant 22 \mathrm{~mm}$, respectively. Because a single colony was not used, to avoid misleading results due to specimens possibly containing a mixture of meticillin-resistant staphylococci ( $S$. aureus and coagulase-negative staphylococci), meticillin-resistant isolates were subcultured on CHROMagar Staph aureus to isolate single colonies and confirm the identification as S. aureus.

Molecular characterization of S. aureus. DNA was extracted as described by Paule et al. (2004). All strains underwent confirmatory
PCR analysis for the mecA gene and for the S. aureus-specific nuc gene (Zhang et al., 2004). In this study, mecA PCR was accepted as the 'gold standard' for meticillin resistance. Multiple-locus variablenumber tandem-repeat fingerprinting (MLVF) was performed using the primer sets described by Sabat et al. (2006). However, to minimize the non-specific bands amplified in the multiplex PCR assay, PCR was performed in three separate reactions containing primer sets for clf $A$ and $c l f B, s d r C D E, s s p A$ and $s p a$, respectively. Amplified products from each reaction were mixed to a $10 \mu \mathrm{l}$ final volume and separated using $1.5 \%$ agarose gels. MLVF patterns were analysed using GelCompar II software (Applied Maths) using the following parameters: optimization, $0.5 \%$; position tolerance, $1.25 \%$. Fingerprinting patterns were clustered, and a dendrogram was generated using the Dice coefficient and UPGMA. Clusters were defined as strains with $\geqslant 70 \%$ genetic relatedness on the dendrogram (Karynski et al., 2008).

Statistical analysis. The $P$ value for differences was calculated with Student's $t$-test. Statistical significance was defined by a $P$ value of less than 0.05 .

\section{RESULTS}

A total of 41 children had cultures positive for S. aureus at both the presurgical outpatient visit (visit 1) and at operation (visit 2), and 33 children had only one culture positive for $S$. aureus. The mean interval between the two sampling times was 13.7 days (range 1-62 days) among children whose paired nasal samples were both positive, and 15.3 days (range 6-52 days) among children who had only one nasal sample positive. Only 8 children, of 360 who had paired nasal and perianal cultures collected, had positive cultures for both nasal and perianal samples.

\section{S. aureus carriage and clonality}

To determine the clonality of $S$. aureus carriage, a total of 738 S. aureus strains were evaluated. The mean number of colonies picked from each swab sample plate was 6 (range 415). Of the total of $125 \mathrm{swab}$ samples positive for $S$. aureus, $91(72.8 \%)$ swabs contained meticillin-sensitive S. aureus (MSSA), 8 (6.4\%) swabs contained MRSA, and 26 (20.8\%) swabs contained MSSA and MRSA simultaneously. Among the MSSA strains, MLVF identified a total of 80 distinct patterns. A dendrogram constructed with MLVF using the UPGMA algorithm with a $70 \%$ similarity index identified 39 clusters, as shown in Fig. 1. Among MRSA strains, a total of 16 distinct MLVF patterns were defined, and 7 clusters were generated, as shown in Fig. 2.

Of the 125 S. aureus positive swab samples, 87 (69.6\%) samples contained only one genotype, whereas 38 (30.4\%) samples contained two or more genetically distinct $S$. aureus isolates. Of these, $32(84.2 \%)$ contained two different genotypes, and $6(15.8 \%)$ contained three different genotypes. The age distributions of children who carried multiple $S$. aureus strains simultaneously and those who carried a single genotype were similar with means of 5.7 and 5.1 years of age, respectively $(P=0.56)$. Of the eight children who had positive cultures for both the paired nasal and perianal samples, six had paired nasal and 


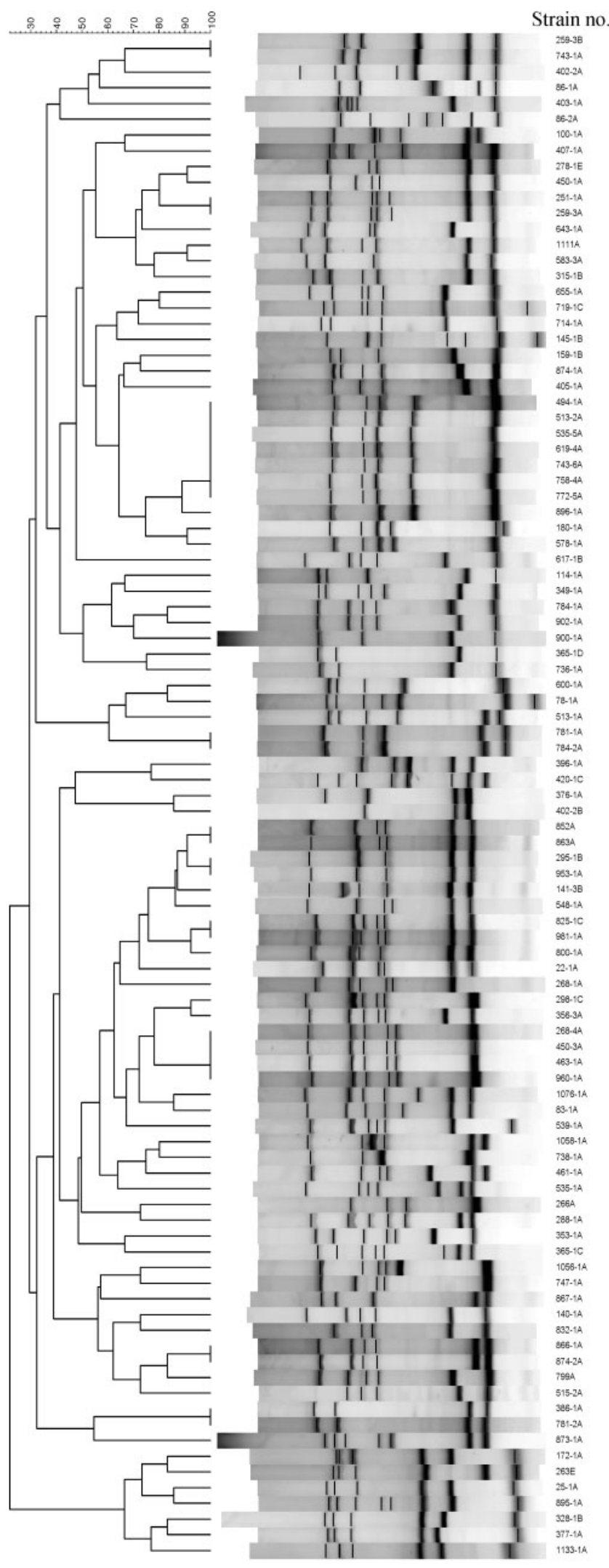

Fig. 1. MLVF dendrogram of selected MSSA strains. The strains represented in the dendrogram included one strain or multiple distinct strains from each individual. perianal samples obtained simultaneously prior to the surgery; the other two had paired samples obtained 6 and 13 days apart, respectively. The results of $S$. aureus genotyping in paired nasal and perianal samples were concordant in only three children.

\section{S. aureus genotypes in successive samples}

The group of 41 children who had paired nasal samples positive for $S$. aureus at visit 1 and 2 was examined to determine the genotypes of successive samples. Nineteen $(46 \%)$ children had identical patterns of S. aureus genotypes in successive nasal samples. Of these, 17 carried a single genotype of MSSA, 1 carried a single genotype of MRSA and 1 carried 2 distinct genotypes of MSSA. The mean interval between the two specimen collections was not significantly different between the groups of children in whom change in genotypes was detected and those without a change in genotypes $(P=0.52)$.

Fig. 3 shows the MLVF patterns of the 22 (54\%) children who harboured different genotypes of $S$. aureus in successive nasal samples. A total of 15 children carried both MSSA and MRSA in the nose on at least 1 occasion. Of these, five had MRSA strains replaced by a distinct MRSA genotype in successive samples, seven had lost the

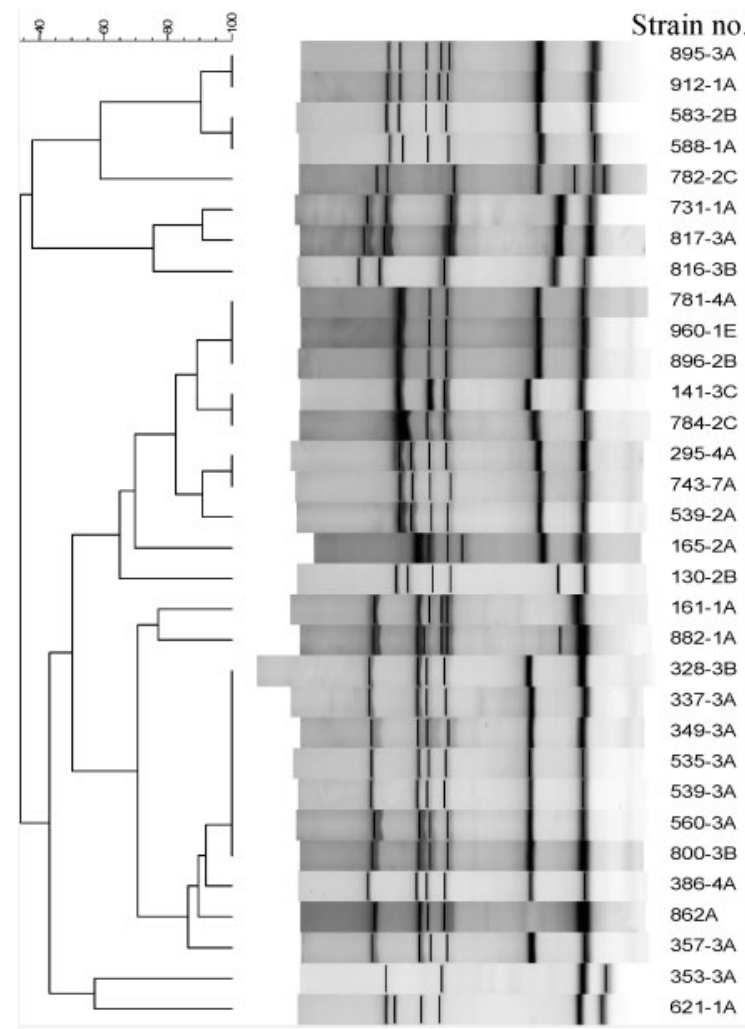

Fig. 2. MLVF dendrogram of selected MRSA strains. The strains represented in the dendrogram included one strain or multiple distinct strains from each individual. 

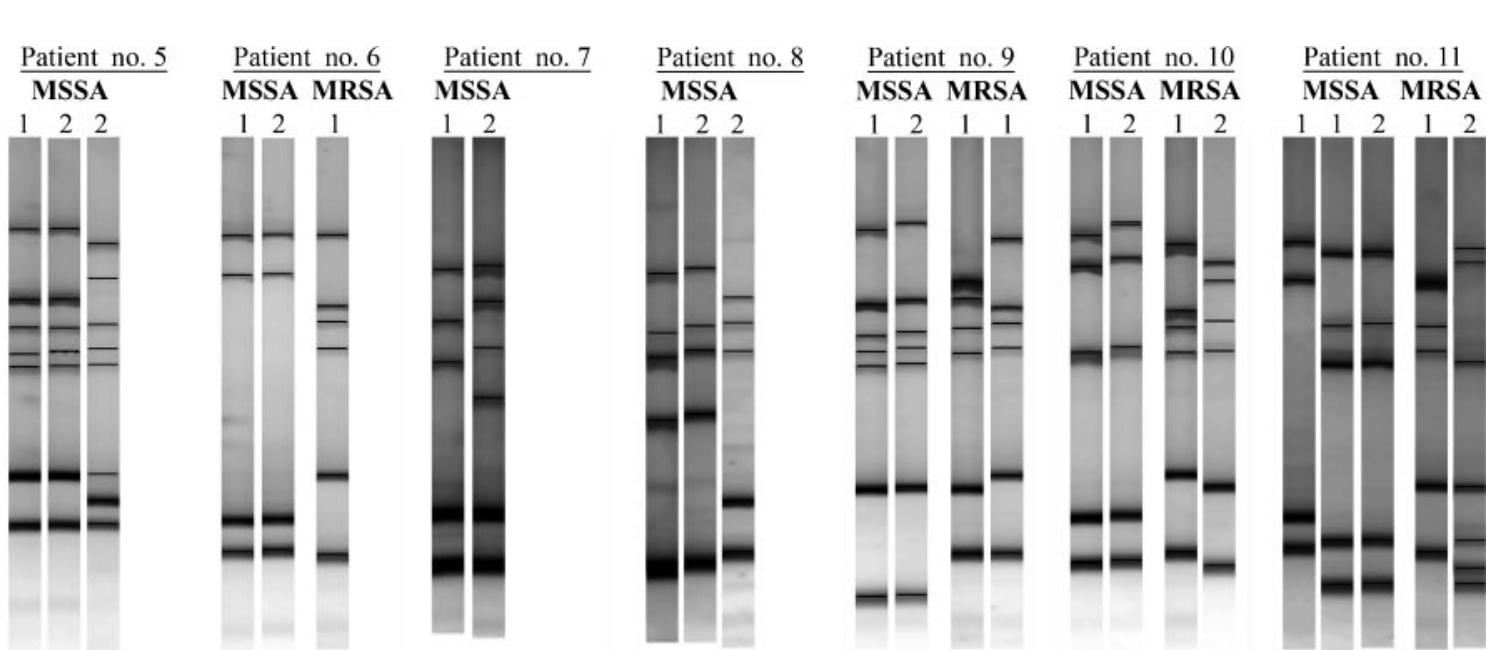

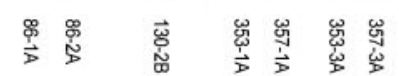

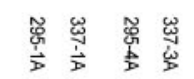

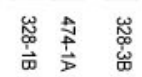

慈兽兽

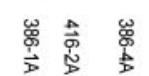

索喜

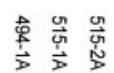

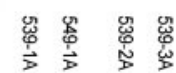

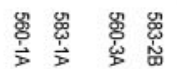

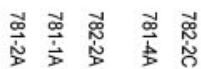

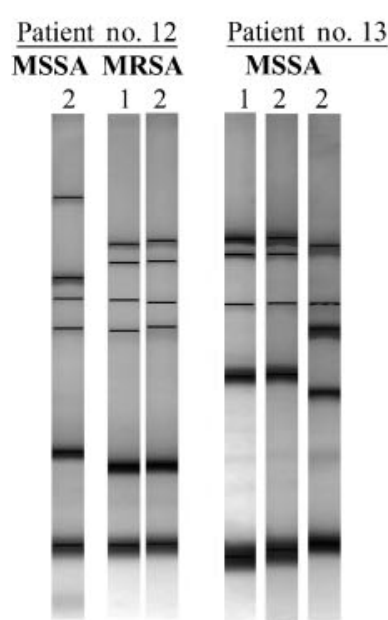

Patient no. 14

Patient no. 15

Patient no. 16 MSSA MSSA MRSA

MSSA MRSA

Patient no. 17 Patient no. 18

Patient no. 19 $12^{2} 2 \quad 11^{1}{ }^{2} 1$
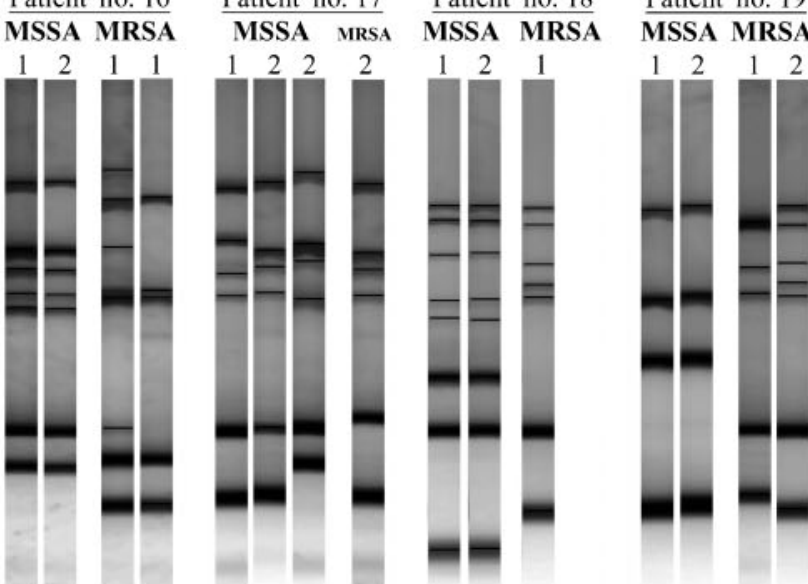

Patient no. 20

Patient no. 2

MSSA MRSA MSSA

Patient no. 22
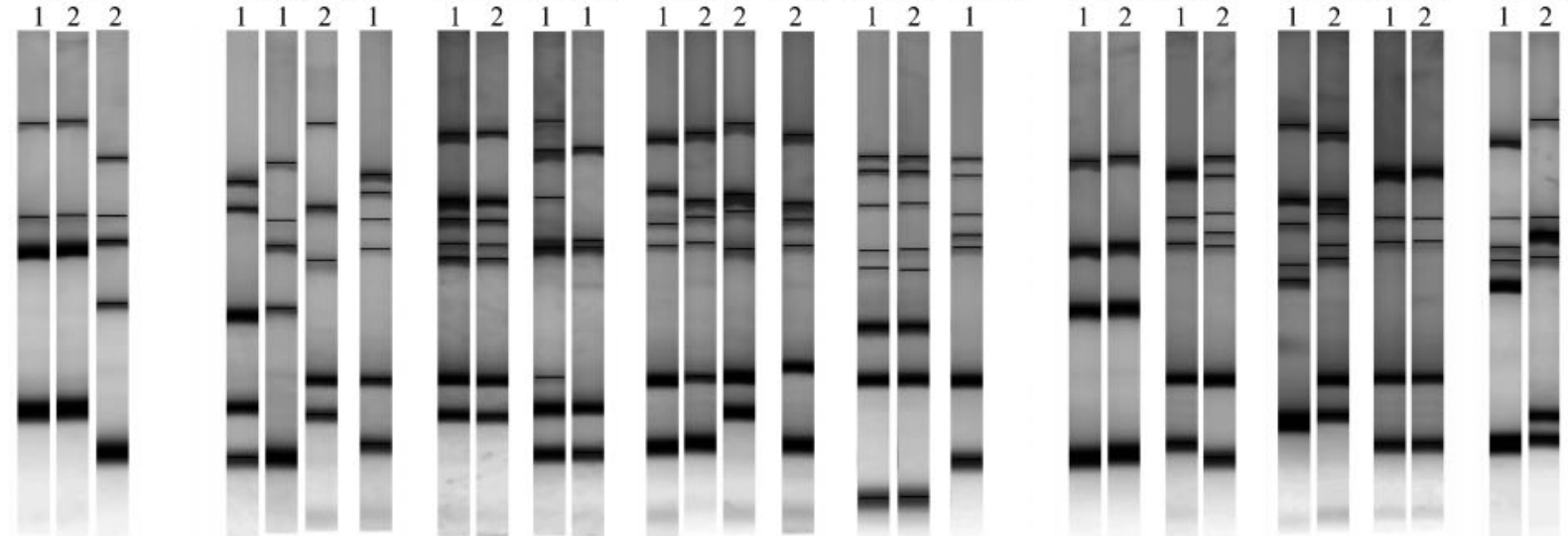

MSSA MRS

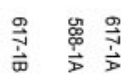

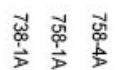

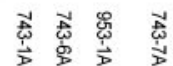

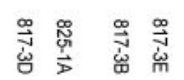

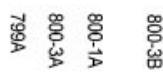

墨兽兽

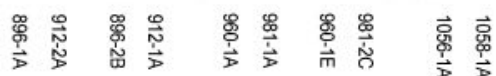

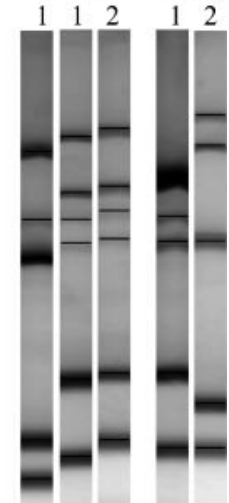

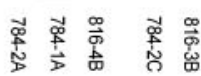

Fig. 3. MLVF of MSSA and MRSA strains in the 22 children who harboured different patterns of $S$. aureus genotypes in successive nasal samples. The numbers 1 and 2 listed at the top of each lane of the MLVF patterns designate the visit number on which the samples were obtained (visit 1 and 2, respectively). The number listed on the bottom of each lane of the MLVF patterns represents the strain number in the study. 
MRSA, two had the same MRSA genotype detected in both samples and one acquired MRSA in the subsequent sample. Despite the findings of either MRSA strain replacement or loss or acquisition of MRSA, $80 \%$ (12 of the 15) apparently had an identical MSSA detected from both initial and subsequent samples.

\section{DISCUSSION}

In this study we investigated the heterogeneity of $S$. aureus colonizing isolates. Of the 125 swab samples that were positive for S. aureus, $30 \%$ of samples contained multiple genotypes. Previously, Cespedes et al. (2005) reported that $\sim 7 \%$ of $S$. aureus colonized individuals carried more than one strain based on their mathematic model analysis. Our results show that polyclonality is more common than originally thought. Based on MLVF pattern analysis, we found that multiple $S$. aureus strains were carried simultaneously by individual children. These strains were genetically unrelated to each other and likely to be the result of multiple strain acquisitions. In children who carried both MSSA and MRSA, we did not detect isogenic MSSA and MRSA strains, i.e. genotypically identical but differing in meticillin susceptibility that could suggest a horizontal gain or loss of the SCCmec element.

Our study provides a picture of the diversity and dynamics of $S$. aureus carriage viewed over a short time span, with a mean interval of 2 weeks between sequential cultures. Our findings are in agreement with other studies (Lebon et al., 2008 ) suggesting that $S$. aureus carriage is a dynamic process involving persistent or transient carriage, loss of strains followed by the acquisition of new strains, and co-carriage of pre-existing strains and new strains. Interestingly, of the 41 children who had paired positive nasal samples, 54\% harboured a different pattern of $S$. aureus genotypes in successive nasal samples. It is unclear why our patients had such a high degree of clonal diversity in successive samples. It is tempting to speculate that the presence of an established strain increases the competitive demands on any new strains, such that they have more difficulty maintaining colonization or are more easily displaced by yet other new strains. Over time, we might expect some equilibrium among competing strains, probably modulated by host immune factors. Our brief period of observation may have been too short a time to arrive at any balance among strains.

The hypothesis that colonization with multiple strains could enhance the potential for horizontal gene transfer was offered as a possible explanation for the observation of acquisition of new virulence determinants (Cespedes et al., 2005). It is also possible that such heterogeneity could place constraints on the bacteria, since bacterial competition is a major ecological interaction and fundamental to the survival of those organisms. Several studies have reported that the carriage rate for S. aureus was significantly lower among subjects with simultaneous carriage of coagulase-negative staphylococci or carriage of corynebacterial species (Lina et al., 2003; Uehara et al., 2000). However, less is known about intraspecies interactions between MSSA and MRSA, and both share the same receptors for adhesion to epithelial cells.

Recently, Dall'Antonia et al. (2005) reported that nasal colonization with MSSA has a protective effect against colonization with MRSA. However, Dall'Antonia and colleagues did not determine the genotypes of those $S$. aureus isolates, and thus the MRSA strain co-carried with MSSA could have been the same $S$. aureus strain with a different degree of phenotypic resistance to meticillin (Kampf, 2005). Here, we were able to show that cocolonization with clonally distinct MSSA and MRSA truly exists. When we examined consecutive and co-colonizing isolates in subjects carrying both MSSA and MRSA, we observed that $56 \%$ had only temporary MRSA cocolonization, and $31 \%$ had MRSA strains replaced by another distinct genotype. In contrast, MSSA strains generally remained constant. Our data may lend support to the idea that colonization with MSSA has a protective effect against colonization with MRSA. Alternatively, the fact that MRSA was not detected from subsequent samples could be due to the transient nature of MRSA carriage.

Several limitations should be addressed. One could argue that the use of MLVF to determine genetic relatedness may have identified isolates as belonging to different strains that would have been identified as belonging to the same strain if PFGE were used to type the S. aureus isolates (Hsu et al., 2006; Stone et al., 2008). The MLVF method is based on the analysis of the five tandem-repeat loci, which are highly polymorphic and rapidly evolving regions. However, several studies have shown that MLVF is a typing method with adequate discriminatory power to differentiate among strains that were not distinguishable by PFGE (Moser et al., 2009; Tenover et al., 2007). Another limitation is the lack of data on other potential factors that may influence the rate of acquisition or the source of clonal diversity, such as overcrowded family conditions or colonized family members. Lastly, the relative numbers of co-colonizing strains were not determined quantitatively to see which strain appeared to be dominant within the population.

In conclusion, our study shows that co-existence of multiple genotypes of $S$. aureus in a single specimen was a common finding. Studies that examine only a single colony, or just a few colonies, from each culture may underestimate the true rate of carriage of multiple genotypes. Although the clinical significance of multiple genotypes remains unclear, there is a possibility that co-colonizing could influence the pathogenicity and virulence of $S$. aureus strains. Understanding this intra-species interplay may reveal the effects of competing clones on their pathogenic potential and yield insights on strategies for disease prevention.

\section{ACKNOWLEDGEMENTS}

This study was supported by the Children's Hospital of Illinois, the Children's Miracle Network and the William H. Albers Professor and 
Chair of the Department of Pediatrics, University of Illinois College of Medicine at Peoria. We thank Lizanne Gray for her technical assistance in picture preparation.

\section{REFERENCES}

Bloemendaal, A. L., Fluit, A. C., Jansen, W. T., Vriens, M. R., Ferry, T., Amorim, J. M., Pascual, A., Stefani, S., Papaparaskevas, J. \& other authors (2009). Colonization with multiple Staphylococcus aureus strains among patients in European intensive care units. Infect Control Hosp Epidemiol 30, 918-920.

Cespedes, C., Said-Salim, B., Miller, M., Lo, S. H., Kreiswirth, B. N., Gordon, R. J., Vavagiakis, P., Klein, R. S. \& Lowy, F. D. (2005). The clonality of Staphylococcus aureus nasal carriage. J Infect Dis 191, 444452.

Coen, P. G., Wilks, M., Dall'antonia, M. \& Millar, M. (2006). Detection of multiple-strain carriers: the value of re-sampling. J Theor Biol 240, 98-103.

Dall'Antonia, M., Coen, P. G., Wilks, M., Whiley, A. \& Millar, M. (2005). Competition between methicillin-sensitive and -resistant Staphylococcus aureus in the anterior nares. J Hosp Infect 61, 62-67.

Goerke, C., Gressinger, M., Endler, K., Breitkopf, C., Wardecki, K., Stern, M., Wolz, C. \& Kahl, B. C. (2007). High phenotypic diversity in infecting but not in colonizing Staphylococcus aureus populations. Environ Microbiol 9, 3134-3142.

Gray, B. M. (2002). Egg-based media for delayed processing of nasopharyngeal swabs in colonization studies of Streptococcus pneumoniae. Eur J Clin Microbiol Infect Dis 21, 666-670.

Herwaldt, L. A. (2003). Staphylococcus aureus nasal carriage and surgical-site infections. Surgery 134, S2-S9.

Hsu, L. Y., Koh, Y. L., Chlebicka, N. L., Tan, T. Y., Krishnan, P., Lin, R. T., Tee, N., Barkham, T. \& Koh, T. H. (2006). Establishment of ST30 as the predominant clonal type among community-associated methicillin-resistant Staphylococcus aureus isolates in Singapore. J Clin Microbiol 44, 1090-1093.

Kampf, G. (2005). What should be done with nasal Staphylococcus aureus carriers? J Hosp Infect 61, 353-354.

Karynski, M., Sabat, A. J., Empel, J. \& Hryniewicz, W. (2008). Molecular surveillance of methicillin-resistant Staphylococcus aureus by multiplelocus variable number tandem repeat fingerprinting (formerly multiple-locus variable number tandem repeat analysis) and spa typing in a hierarchic approach. Diagn Microbiol Infect Dis 62, 255-262.

Keene, A., Vavagiakis, P., Lee, M. H., Finnerty, K., Nicolls, D., Cespedes, C., Quagliarello, B., Chiasson, M. A., Chong, D. \& Lowy, F. D. (2005). Staphylococcus aureus colonization and the risk of infection in critically ill patients. Infect Control Hosp Epidemiol 26, 622-628.

Kreft, B., Eckstein, S., Kahl, A., Frei, U., Witte, W. \& Trautmann, M. (2001). Clinical and genetic analysis of Staphylococcus aureus nasal colonisation and exit-site infection in patients undergoing peritoneal dialysis. Eur J Clin Microbiol Infect Dis 20, 734-737.

Lebon, A., Labout, J. A. M., Verbrugh, H. A., Jaddoe, V. W. V., Hofman, A., Van Wamel, W., Moll, H. A. \& Van Belkum, A. (2008). Dynamics and determinants of Staphylococcus aureus carriage in infancy: the Generation R Study. J Clin Microbiol 46, 3517-3521.
Lim, M. S., Marshall, C. L. \& Spelman, D. (2006). Carriage of multiple subtypes of methicillin-resistant Staphylococcus aureus by intensive care unit patients. Infect Control Hosp Epidemiol 27, 1063-1067.

Lina, G., Boutite, F., Tristan, A., Bes, M., Etienne, J. \& Vandenesch, F. (2003). Bacterial competition for human nasal cavity colonization: role of staphylococcal agr alleles. Appl Environ Microbiol 69, 18-23.

Lomholt, H., Andersen, K. E. \& Kilian, M. (2005). Staphylococcus aureus clonal dynamics and virulence factors in children with atopic dermatitis. J Invest Dermatol 125, 977-982.

Moser, S. A., Box, M. J., Patel, M., Amaya, M., Schelonka, R. \& Waites, K. B. (2009). Multiple-locus variable-number tandem-repeat analysis of meticillin-resistant Staphylococcus aureus discriminates within USA pulsed-field gel electrophoresis types. J Hosp Infect 71, 333-339.

Niven, D. J., Laupland, K. B., Gregson, D. B., Church, D. L. \& the S. aureus Screening Initiative Group (2009). Epidemiology of Staphylococcus aureus nasal colonization and influence on outcome in the critically ill. J Crit Care 24, 583-589.

Paule, S. M., Pasquariello, A. C., Hacek, D. M., Fisher, A. G., Thomson, R. B., Jr, Kaul, K. L. \& Peterson, L. R. (2004). Direct detection of Staphylococcus aureus from adult and neonate nasal swab specimens using real-time polymerase chain reaction. J Mol Diagn 6, 191-196.

Sabat, A., Malachowa, N., Miedzobrodzki, J. \& Hryniewicz, W. (2006). Comparison of PCR-based methods for typing Staphylococcus aureus isolates. J Clin Microbiol 44, 3804-3807.

Stone, N. D., Lewis, D. R., Lowery, H. K., Darrow, L. A., Kroll, C. M., Gaynes, R. P., Jernigan, J. A., McGowan, J. E., Jr, Tenover, F. C. \& Richards, C. L., Jr (2008). Importance of bacterial burden among methicillin-resistant Staphylococcus aureus carriers in a long-term care facility. Infect Control Hosp Epidemiol 29, 143-148.

Tenover, F. C., Vaughn, R. R., McDougal, L. K., Fosheim, G. E. \& McGowan, J. E., Jr (2007). Multiple-locus variable-number tandemrepeat assay analysis of methicillin-resistant Staphylococcus aureus strains. J Clin Microbiol 45, 2215-2219.

Uehara, Y., Nakama, H., Agematsu, K., Uchida, M., Kawakami, Y., Abdul Fattah, A. S. \& Maruchi, N. (2000). Bacterial interference among nasal inhabitants: eradication of Staphylococcus aureus from nasal cavities by artificial implantation of Corynebacterium sp. J Hosp Infect 44, 127-133.

Vegunta, R. K., Gray, B., Wallace, L. J., Mongkolrattanothai, K., Mankin, P., Stanfill, A. B. \& Pearl, R. H. (2009). A prospective study of methicillin-resistant Staphylococcus aureus colonization in children scheduled for elective surgery. J Pediatr Surg 44, 1197-1200.

Von Eiff, C., Becker, K., Machka, K., Stammer, H. \& Peters, G. (2001). Nasal carriage as a source of Staphylococcus aureus bacteremia. N Engl J Med 344, 11-16.

Wenzel, R. P. \& Perl, T. M. (1995). The significance of nasal carriage of Staphylococcus aureus and the incidence of postoperative wound infection. J Hosp Infect 31, 13-24.

Zhang, K., Sparling, J., Chow, B. L., Elsayed, S., Hussain, Z., Church, D. L., Gregson, D. B., Louie, T. \& Conly, J. M. (2004). New quadriplex PCR assay for detection of methicillin and mupirocin resistance and simultaneous discrimination of Staphylococcus aureus from coagulasenegative staphylococci. J Clin Microbiol 42, 4947-4955. 